\title{
Haben die NRO ein Legitimitätsproblem?
}

Peter Niggli and André Rothenbühler

\section{(2) OpenEdition}

\section{Journals}

Electronic version

URL: http://journals.openedition.org/sjep/494

DOI: $10.4000 /$ sjep.494

ISSN: 1663-9677

\section{Publisher}

Institut de hautes études internationales et du développement

\section{Printed version}

Date of publication: 1 novembre 2004

Number of pages: $27-33$

ISBN: 2-88247-057-6

ISSN: $1660-5926$

\section{Electronic reference}

Peter Niggli und André Rothenbühler, « Haben die NRO ein Legitimitätsproblem? », Schweizerisches Jahrbuch für Entwicklungspolitik [Online], 23-2 | 2004, Online erschienen am: 17 Juni 2010, abgerufen am 07 September 2020. URL : http://journals.openedition.org/sjep/494 ; DOI : https://doi.org/ 10.4000/sjep.494 


\title{
Haben die NRO ein Legitimitätsproblem?'
}

\author{
Peter Niggli* und André Rothenbühler**
}

\begin{abstract}
ür viele Regierungen, internationale Organisationen und global tätige Unternehmen ist es heute ein Muss, Nichtregierungsorganisationen (NRO) in ihre Arbeit einzubeziehen. Gleichzeitig äussern dieselben Institutionen gegenüber NRO vermehrt Kritik und bezweifeln deren Legitimität. Sie hätten die NRO gerne etwas ,handzahmer" und doch leicht oppositionell.
\end{abstract}

Seit den ersten Demonstrationen gegen internationale Regierungskonferenzen schimpfen Globalisierungsverfechter, die NRO, welche sich als Sittenwächter der internationalen Politik aufspielten, sprächen nur für sich selbst. Es sei absurd, dass solche Organisationen multilaterale Entscheidungen als undemokratisch kritisieren. Immerhin seien die angegriffenen Regierungen durch Wahlen legitimiert, während die „NRO-Funktionäre“ durch niemanden, und schon gar nicht durch die Zivilgesellschaft gewählt seien.

Der Vorwurf mangelnder Legitimität durchzieht seither die öffentliche Diskussion über Nichtregierungsorganisationen. Unüberhörbar ertönt der Ruf, diese irgendeiner Form von Regulierung zu unterwerfen. Diesbezüglich sind zwei konkrete Initiativen von Bedeutung.

\section{Plädoyers für mehr oder weniger NRO-Einfluss}

UNO-Generalsekretär Kofi Annan setzte im Sommer 2003 ein Panel of Eminent Persons on Civil Society and UN Relationships unter dem Vorsitz des ehemaligen brasilianischen Präsidenten Fernando Henrique Cardoso ein ${ }^{2}$. Ziel war es, „die Vertretung der Zivilgesellschaft“" in der internationalen Politik und vor allem in der UNO zu ,vergrössern“. Dazu sollte der Ausschuss unter anderem ein Reihe von „rechtlichen Leitlinien“ vorlegen, an denen sich alle NRO messen lassen müssten. Gleichzeitig solle man aber anerkennen, dass die NRO für ihre Arbeit eine ,gewisse Freiheit“ bräuchten, so Panel-Koordinator Kevin Kennedy. Der Ausschuss arbeitete mit der Annahme, dass NRO die Zivilgesellschaft auch als „Nichtgewählte“ vertreten dürfen, sofern sie sich den Richtlinien der UNO anpassen.

Der Schlussbericht, welcher Kofi Annan im Juni 2004 übergeben wurde, umgeht die heiklen Fragen. Der Bericht plädiert dafür, die NRO vermehrt in die Arbeit der UNO-Instanzen einzubeziehen, ihre Akkreditierung zu vereinheit-

1 Dieser Text ist die Überarbeitung eines im Dezember 2003 in Global+ veröffentlichten Artikels.

* Geschäftsleiter der Arbeitsgemeinschaft Swissaid/Fastenopfer/Brot für alle/Helvetas/Caritas/HEKS und Leiter des Bereichs Entwicklungspolitik. Zur Arbeitsgemeinschaft der Hilfswerke siehe Rahmentext am Schluss des Artikels.

** Mitarbeiter der Erklärung von Bern, Journalist der Presseagentur InfoSüd, Bern.

2 „We the Peoples: Civil Society, the United Nations and Global Governance." Report of the Panel of Eminent Persons on United Nations-Civil Society Relations, Juni 2004 : <www.un.org/reform/panel.htm>. 
lichen und zu vereinfachen, und mehr NRO aus dem Süden zu berücksichtigen. Der Bericht bemüht sich, diesbezügliche Vorbehalte der Regierungen von Entwicklungsländern auszuräumen. Auf das Thema der „rechtlichen Leitlinien“, an denen sich alle NRO messen lassen müssten, geht der Bericht jedoch nicht ein.

Ebenfalls im Sommer 2003 lancierte das American Enterprise Institute - ein Think Tank der Bush-Regierung - zusammen mit verwandten Institutionen das Projekt NGOWatch${ }^{3}$. „Viele NRO“ seien „von ihrem ursprünglichen Mandat abgekommen und [hätten] regierungsähnliche Rollen übernommen“, klagten die Initianten. „Das aussergewöhnliche Wachstum anwaltschaftlicher NRO hat das Potential, die Souveränität konstitutioneller Demokratien zu unterminieren.“ Die NRO bevorzugten eine Weltordnung der Global Governance oder Weltregierung auf Kosten der Souveränität demokratischer Nationalstaaten. Darin würden sie von der UNO unterstützt. NGOWatch will die US-Regierung und multinationale Unternehmen über die Gefahren der Zusammenarbeit mit NRO aufklären und von letzteren mehr Transparenz und Rechenschaftspflicht einfordern. Sie sollen ihre anwaltschaftliche Einmischung aufgeben und auf den Pfad karitativer Arbeit zurückkehren.

Nach der Website zu urteilen, ist NGOWatch allerdings nicht recht vom Fleck gekommen. Der Impetus, den grossartigen Sieg im Irak nun auch für ein Generalaufräumen an der NRO-Front zu benutzen, hat in den Widrigkeiten, mit denen die Bush-Administration im Wahljahr zu kämpfen hatte, an Schwung verloren. Es gelang dem Projekt vorderhand auch nicht, eine grössere internationale Ausstrahlung zu entwickeln.

\section{Amtlich bewilligte NRO}

Es ist eine zentrale Anforderung, dass Regierungen, welche im Namen ihrer Länder agieren, gewählt sein sollten. Daraus folgt jedoch nicht, dass auch NRO gewählt sein müssen, um Zugang zu Regierungen zu haben und diese kritisieren zu dürfen. Aktivitäten und Kritik von Nichtregierungsorganisationen werden durch die politischen Grundrechte einer demokratisch verfassten Öffentlichkeit ausreichend legitimiert. Die Öffentlichkeit entscheidet, ob Kritik und Vorschläge der NRO etwas taugen. Aber sogar, wenn die Öffentlichkeit die politischen Auffassungen der NRO mehrheitlich ablehnt, würde das an deren Legitimität, zu handeln, wie sie es tun, kein Jota ändern. Bekanntlich erfüllt sich die Substanz demokratischer Grundrechte erst dann, wenn Minderheitsauffassungen den Respekt der Mehrheit und den Schutz des Gesetzes geniessen.

Im Übrigen beansprucht jedes global tätige Unternehmen direkten Zugang zu den Regierungen. Wirtschaftslobbyisten begleiten seit Jahrzehnten jede internationale Verhandlung und beeinflussen Verhandlungsagenda und -gegenstand. Wichtige Regierungsabkommen auf regionaler oder internationaler Ebene sind auf Initiative von Konzernen und Unternehmensvereinigungen entstanden und haben deren Begehren weitgehend übernommen: Den Anstoss und Entwurf für den EU-Binnenmarkt hat der European Roundtable of Industrialists (ERT) geliefert. Anstoss und Entwurf für das Dienstleistungsabkommen (GATS) der Welthandelsorganisation kamen von der Vereinigung der amerikanischen

3 NGOWatch: <www.NGOWatch.org>. 
Dienstleistungsfirmen. Die Konzerne und Wirtschaftsvereinigungen wurden aber für ihre Einflussnahme weder durch die Belegschaften, noch durch die Bevölkerungen ihrer „Heimatländer“ gewählt oder beauftragt.

Zur Verwirrung der Diskussion trägt allerdings bei, dass einige NRO versuchen, ihre Legitimität ohne Rekurs auf die Grundrechte zu verteidigen. Sie postulieren, die Zivilgesellschaft zu ,vertreten“, und beanspruchen eine Repräsentationsfunktion, die - wenn wir von Gewerkschaften oder Bauernorganisationen absehen praktisch keine NRO hat. Es wundert nicht, dass sie mit diesem Anspruch den Einwand, niemand habe sie als Vertreter der Zivilgesellschaft gewählt, nicht erwidern können. Unseres Erachtens besteht denn auch das Wesen vieler NRO nicht darin, jemanden zu vertreten, sondern Gruppen und Problemen Gehör zu verschaffen, die es gar nicht zur Repräsentation ihrer Interessen bringen oder die im Streit der tonangebenden Interessen unterzugehen drohen.

Die NRO-Kritiker gehen aus nahe liegenden Gründen nicht auf das Thema der politischen Grundrechte ein. Stattdessen fordern sie neue Instrumente, um die angebliche Legitimitätslücke zu füllen. So schlug zum Beispiel das regierungsnahe britische Think Tank Foreign Policy Center vor, staatliche Verhaltensregeln für NRO zu erlassen und eine Aufsichtsbehörde einzusetzen, welche die NRO zertifizieren soll. Solche Vorschläge laufen darauf hinaus, NRO durch staatliche Anerkennung zu legitimieren und ihre kritische Rolle durch Richtlinien zu kanalisieren. Mit der Lizenzierung erhielten die Behörden auch einen Hebel, die „vernünftigen“ NRO von den „radikalen“ zu trennen.

\section{Probleme der Legitimität oder der Geschäftsführung?}

Es gibt einzelne Stimmen, die auf die Abartigkeit der Legitimitätsdiskussion hinweisen. So kommentierte die New York Times die NGOWatch-Initiative: „Natürlich stehen die politischen Ansichten von NRO zur freien Debatte und Kritik; Rechenschaftspflicht und Transparenz sollten sich aber um Praktiken, und nicht um Politik drehen."

Damit weist die Zeitung darauf hin, dass NRO auch Unternehmen sind, welche Dienstleistungen erbringen, von Spendengeldern leben und oft Staatssubventionen erhalten. Hinsichtlich ihrer Geschäftsführung darf von ihnen Transparenz und Rechenschaftspflicht verlangt werden. Da die meisten steuerbefreit sind, müssen sie ohnehin staatliche Auflagen an ihr Geschäftsgebaren erfüllen. In vielen Ländern, darunter die Schweiz, unterwerfen sich NRO freiwilligen Richtlinien der Mittelverwendung, die vor allem der Sicherung des Spendenzwecks dienen sollen. Gegenwärtig sind ausserdem vielerorts neue Initiativen zur Stärkung der Corporate Governance von NRO im Gang, welche der Professionalisierung der Branche und ihrem Wachstum Rechnung tragen sollen.

Wenn in der Legitimitätsdiskussion über Richtlinien für NRO gesprochen wird, bleibt es oft unklar, was für welche gemeint sind - Richtlinien für ihr politisches Verhalten oder Richtlinien für ihre „gute Geschäftsführung“. Gute Geschäftsführung einzufordern, ist selbstverständlich vertretbar. Geht es um politisches Wohlverhalten, wird es problematisch. 
Die Diskussion um die Legitimität von NRO begann, weil vor allem Regierungsvertreter von Industrieländern und die global tätige Wirtschaft die globalisierungskritische Bewegung als sehr lästig empfinden und die bekannten Umwelt-, Entwicklungs- und Menschenrechts-NRO, welche eine hohe öffentliche Glaubwürdigkeit geniessen, gerne völlig aus der Bewegung herauslösen möchten. Die gleichen Instanzen suchen aber ihre Zusammenarbeit mit eben diesen NRO zu systematisieren, da sie erwarten, multilaterales Handeln dadurch besser legitimieren zu können.

Bei multilateralen Verhandlungen und den daraus resultierenden Verträgen und internationalen Institutionen fungieren die beteiligten Regierungen als Legislative, Exekutive und manchmal auch Judikative in einem. Es gibt keine checks and balances wie im demokratisch verfassten Nationalstaat. Die Resultate solcher Verhandlungen brechen ausserdem - im wirtschaftlichen Bereich - nationales Recht, ohne dass nationale Öffentlichkeiten, Interessengruppen und Parlamente ein substantielles Beratungs- und Entscheidungsrecht wahrnehmen könnten. Die Tatsache, dass die Regierungen auf nationaler Ebene gewählt sind, legitimiert ihre umfangreiche gesetzgeberische Tätigkeit auf internationaler Ebene nur ungenügend. Das ist das Demokratiedefizit der internationalen Politik. Es hat entscheidend zur Entstehung der globalisierungskritischen Bewegung beigetragen.

Wenn nun NRO internationale Regierungskonferenzen begleiten dürfen, Zugang zu ausgewählten Informationen erhalten und zu den anstehenden Geschäften angehört werden, hilft dies mit, das Demokratiedefizit zu „kaschieren“. Falls NRO vor der anwesenden „Weltöffentlichkeit“ die Regierungen sogar kritisieren und alternative Forderungen erheben, stärkt das den Anschein offener demokratischer Auseinandersetzung. Wenn eine Regierung danach zu Hause einer Parlamentskommission sagt, sie habe zu den wichtigen internationalen Geschäften die NRO konsultiert, suggeriert sie, soweit wie möglich alle Gesichtspunkte der Opposition berücksichtigt zu haben. Das ist meistens nicht der Fall. Man sieht jedoch: Kritische NRO sind nicht nur lästig, weshalb die Gegenspieler ihre Legitimität anzweifeln, sondern auch nützlich.

Die NRO als „dritter Stand“?

Seit zwei, drei Jahren wirbt die UNO - und sie wird darin durch die Regierungen der Industrieländer unterstützt - systematisch für tripartite Kooperationen zwischen Regierungen, Zivilgesellschaft und Wirtschaft. Das Cardoso-Panel arbeitete mit der Annahme, dass es drei internationale Akteure gäbe - Regierungen, Zivilgesellschaft und Privatsektor -, deren Zusammenspiel zu definieren sei und die künftige Global Governance ausmache. Angestrebt wird ein korporatistisches Konstrukt, in dem die NRO sozusagen als Vertreter des „,dritten Standes“ fungieren.

Michael Edwards, Leiter der Governance and Civil Society Unit der Ford Foundation, einer wichtigen Finanzierungsquelle für amerikanische und internationale NRO, hat das korporatistische Modell in einem Buch weitergetrieben ${ }^{4}$. Sei-

4 Michael Edwards: NGO Rights and Responsibilities. A New Deal for Global Governance. Foreign Policy Center, 2000. 
nes Erachtens sollte „das Recht der NRO, sich öffentlich einzumischen“, durch eine Reihe von „Compacts“ (Abkommen) zwischen Regierungen, Unternehmen und NRO-Netzwerken ,strukturiert“ werden. Dies soll von unten nach oben, also von der regionalen über die nationale bis zur internationalen Ebene geschehen. Diese Abkommen würden die „Rollen und Verantwortlichkeiten“ der Akteure im Hinblick auf bestimmte Themen und Institutionen regeln. Edwards sieht den Vorteil seines Modells darin, dass NRO die nationale Ebene (bzw. die politische Einigung auf nationaler Ebene) nicht mehr umgehen und Konflikte nicht mehr direkt auf die internationale Ebene tragen könnten. Er zitiert als Beispiel den Streit um das Wasserkraftprojekt Arun-III in Nepal, welches die Weltbank mitfinanzieren wollte, und das an einer internationalen Kampagne von NRO gescheitert sei. Hier habe man die mühsame Arbeit des nationalen coalition building einfach umgangen und lokalen Arun-III-Verfechtern zuwenig Gehör geschenkt.

Das Edwardssche Modell würde die Mächtigen stärken und den Einfluss der NRO schwächen. Es zeigt zudem die Problematik, welche dem korporatistischen Ansatz für die Global Governance innewohnt. Wird das Konstrukt auf die nationale Ebene übertragen, steht es sofort im Widerspruch zu vorhandenen demokratischen Mechanismen. Man stelle sich einen „Compact“ zwischen Bundesrat, economiesuisse und NRO für die Schweiz vor. Wie soll ein solcher zu den Parlamenten aller Ebenen, den Gemeinde- und Kantonsexekutiven, den politischen Parteien und den direktdemokratischen Instrumenten stehen? Eine Überordnung triebe die Schweizerinnen und Schweizer zum Aufstand, eine Unterordnung hingegen würde ein solches Abkommen sinnlos machen. Sowohl Wirtschaft wie NRO fahren auf nationaler Ebene besser, wenn sie ohne korporatistisches Gebilde direkt die demokratischen Mechanismen nutzen. Es gibt keinen Grund, weshalb das Modell in Entwicklungsländern besser und demokratischer sein soll. Der tripartite Korporatismus ist nur auf internationaler Ebene oder in autoritären Staaten denkbar, dort also, wo es keine Demokratie gibt.

Kurz, es gibt gute Gründe, weshalb NRO den Verlockungen einer korporatistisch strukturierten Global Governance nicht kritiklos erliegen sollten. Natürlich ist es besser, wenn die NRO für die Fragen der Global Governance miteinbezogen werden und das Feld nicht allein den Vertretern globaler Konzerne überlassen, die sich ohnehin seit langem und ganz selbstverständlich beteiligen. Unseres Erachtens ist dies das wichtigste Argument, mit welchem die NRO ihr Begehren, in Fragen der Global Governance angehört zu werden, begründen sollten. Ihr Einbezug macht jedoch die internationale Politik nicht demokratischer. Sie sollten deshalb weiterhin das Demokratiedefizit der Global Governance kritisieren und den Transfer politischer Kompetenzen von der nationalen auf die internationale Ebene mit grosser Skepsis begleiten. 


\section{Die Arbeitsgemeinschaft der Hilfswerke: eine Lobbying- und Anwaltschaftsfunktion}

Die Arbeitsgemeinschaft der Hilfswerke wird von sechs grossen Hilfswerken der Schweiz getragen und finanziert. Zwei davon sind mit dem schweizerischen Katholizismus liiert (Fastenopfer und Caritas), zwei mit den protestantischen Kirchen (Brot für alle und HEKS), während zwei weltanschaulich und religiös nicht gebunden sind (Swissaid und Helvetas). Die Arbeitsgemeinschaft wurde 1971 von Swissaid, Brot für alle und Fastenopfer gegründet. Die weiteren Mitglieder stiessen später dazu, zuletzt das HEKS im Jahre 2003.

Die Arbeitsgemeinschaft der Hilfswerke ist kein Dachverband und erfült auch keine Koordinationsfunktion für ihre Mitglieder. Vielmehr haben diese das Lobbying und die anwaltschaftliche Öffentlichkeitsarbeit in ausgewählten entwicklungspolitischen Fragen an das Gemeinschaftsunternehmen „Arbeitsgemeinschaft" delegiert.

\section{Handlungsfelder}

\section{Entwicklungszusammenarbeit und -politik}

1. Die Theorie und Praxis sowie die Zielkonflikte der schweizerischen Entwicklungszusammenarbeit und humanitären Hilfe.

2. Die Zielkonflikte bei südpolitischen Interventionen verschiedener Regierungsstellen, die als so genanntes Kohärenzproblem bekannt sind.

3. Die Theorie und Praxis multilateraler Entwicklungszusammenarbeit, insbesondere die Politik der Schweiz in der Weltbank und anderen vergleichbaren Agenturen.

4. Die internationale und schweizerische Entschuldungspolitik.

\section{Internationale Wirtschaftspolitik}

5. Die internationale Finanzpolitik, die schweizerische Politik im Internationalen Währungsfonds (IWF) und entwicklungspolitisch bedeutsame Aspekte des Schweizer Finanzplatzes.

6. Die internationale Handelspolitik, die Aussenwirtschaftspolitik der Schweiz und deren Politik in der Welthandelsorganisation (WTO).

7. Die Praxis von schweizerischen multinationalen Unternehmen in Entwicklungsländern sowie die internationale Politik und die Nord-Süd-Politik ihrer nationalen und internationalen Verbände.

\section{Global Governance}

8. Die internationale Umweltpolitik und entsprechende Positionen der Schweiz.

9. Weitere entwicklungspolitisch bedeutsame Probleme der Global Governance (UNO, UNO-Sondergipfel, G-7, die Weltpolitik der USA und anderer Grossmächte) und die entsprechende schweizerische Politik.

\section{Ziele}

1. Die Arbeitsgemeinschaft der Hilfswerke will bewirken, dass das Parlament den Bundesratsentscheid, den Kohäsionsbeitrag an die EU über die Entwicklungsbudgets zu finanzieren, korrigiert oder zumindest abschwächt.

Im Jahr 2004 beschloss der Bundesrat, den Kohäsionsbeitrag an die EU in der Höhe von 1 Milliarde Franken über fünf Jahre voll zu Lasten des Wirtschafts- und des Aussenministeriums zu kompensieren. Beide Ministerien werden dies auf Kosten der Entwicklungszusammenarbeit tun. Damit würden die Nutzniesser der Entwicklungshilfe - die ärmsten Länder - die Kosten der Bilateralen II, darunter den Schutz des Steuerfluchtgeheimnisses bezahlen, ohne am Nutzen zu partizipieren, den die Schweiz aus den neuen Verträgen mit der EU zieht. Dieses Vorgehen finden auch wertkonservative Kreise im Parlament unethisch. Wird der Bundesratsentscheid nicht korrigiert, würde die schweizerische Entwicklungshilfe um rund 20 Prozent gekürzt werden, obwohl der Bundesrat immer noch behauptet, er wolle die Hilfe bis zum Jahr 2010 auf 0,4 Prozent des Bruttosozialprodukts erhöhen.

2. Das internationale System der Steuerhinterziehung durch die mobilsten Akteure der Weltwirtschaft, das allen Staaten schadet und den Entwicklungsländern Steuereinnahmen von jährlich rund 50 Milliarden Dollar entzieht, soll durch multilaterale Organisationen problematisiert und drastisch eingeschränkt 
werden. Der staatliche Schutz für ausländische Steuerhinterzieher durch die Schweiz - irreführenderweise „Bankgeheimnis“ genannt - soll im Verlauf der nächsten zehn Jahre gelockert und abgebaut werden. Zu diesem Zweck hat die Arbeitsgemeinschaft das internationale NRO-Netzwerk namens Tax Justice Network ins Leben gerufen. Dieses geht gegen die Off-Shore-Praktiken von England, Frankreich, Belgien und der Schweiz vor und versucht, bei der OECD, der UNO und anderen interessierten internationalen Organisationen ein koordiniertes Eindämmen solcher Praktiken anzustossen.

3. Anstelle einer "umfassenden" Verhandlungsrunde ist in der Welthandelsorganisation (WTO) eine Entwicklungsrunde anzustreben. Diese soll die einseitige Benachteiligung der Entwicklungsländer durch die bestehenden Abkommen korrigieren und neue Regeln verabschieden, welche den wirtschaftspolitischen Spielraum der einzelnen Entwicklungsländer wieder herstellen und schützen. Der Bundesrat soll bewegt werden, eine solche Entwicklungsrunde zu befürworten. Weiter soll er unter den Industrieländern die Bereitschaft fördern, den Entwicklungsländern einseitige Konzessionen ohne "gleichwertige" Gegenleistungen zu machen. Die Arbeitsgemeinschaft begleitet seit der WTO-Ministerkonferenz in Seattle die WTO-Politik des Bundesrats in einer losen Allianz mit den Gewerkschaften, sämtlichen Bauernverbänden, grossen Umweltorganisationen und Entwicklungsorganisationen. Die Allianz vereinigt diejenigen, deren Interessen oder ideelle Anliegen durch den neuen Handelsliberalisierungsschub, den die Industrieländer seit 1999 gegen jegliche Widerstände durchsetzen wollen, geschädigt würden.

4. Der Zugang zu Trinkwasser als Menschenrecht soll durch eine internationale Wasserkonvention kodifiziert werden. Die Arbeitsgemeinschaft der Hilfswerke hat dazu ein internationales NRO-Netzwerk ins Leben gerufen, das geeignete Staaten dazu bewegen will, die Initiative zu ergreifen. Sie glaubte anfänglich, dass sich vor allem auch die Schweiz für eine Wasserkonvention einsetzen würde. Im Jahr 2001 hatte das Parlament ein entsprechendes Postulat an den Bundesrat überwiesen. Dieser nahm den Vorstoss entgegen und versprach, sich für das Zustandekommen einer Konvention einzusetzen. Das Anliegen ist seither in der Verwaltung versandet. Dort sträuben sich Beamte dagegen, weil sie eine Konvention für nicht dringlich erachten und befürchten, eine solche richte sich gegen die Privatisierung von Wasserversorgungen im Süden. Sie glauben, eine Trinkwasserversorgung für alle könne nur durch massive Investitionen des Privatsektors zustande kommen, und übersehen, dass private Unternehmen am liebsten dann in Wasserversorgungen investieren, wenn staatliche Gelder und Garantien ihre Risiken voll decken. Um ihrem Anliegen Nachdruck zu verleihen, hat die Arbeitsgemeinschaft im Jahr 2004 die Initiative zur Bildung eines internationalen Netzwerkes von grossen NRO ergriffen. Unter anderem beteiligen sich das deutsche Hilfswerk Brot für die Welt, der Council of Canadians und WeDo (das internationale Netzwerk Frauen und Entwicklung). Diese "Friends of a Convention“ werben für eine Wasserkonvention und suchen die Zusammenarbeit mit einzelnen Staaten, UNO-Organisationen und anderen interessierten Institutionen. Die Arbeitsgemeinschaft der Hilfswerke wird dann wieder auf die Schweizer Regierung zugehen, wenn sich andere Regierungen für eine Konvention stark zu machen beginnen. 